\title{
How important is the Family? Alpha nuclear potentials and $p$-process nucleosynthesis *
}

\author{
A. Ornelas ${ }^{\dagger a}$, D. Galaviz ${ }^{a}$, Zs. Fülöp ${ }^{b}$, Gy. Gyürky $^{b}$, G. Kiss ${ }^{b}$, Zs. Máté ${ }^{b}$, P. Mohr ${ }^{c}, \mathbf{T}$. \\ Rauscher $^{d}$, E. Somorjai ${ }^{b}$, K. Sonnabend ${ }^{e}$ and A. Zilges ${ }^{f}$ \\ ${ }^{a}$ Centro de Física Nuclear, University of Lisbon, 1649-003 Lisbon, Portugal \\ ${ }^{b}$ ATOMKI, H-4001 Debrecen, POB. 51, Hungary \\ ${ }^{c}$ Diakonie-Klinikum, D-74523 Schwäbisch Hall, Germany \\ ${ }^{d}$ Departement für Physik und Astronomie, Universität Basel, CH-4056 Basel, Switzerland \\ ${ }^{e}$ Institut für Kernphysik, TU-Darmstadt, D-64289 Darmstadt, Germany \\ ${ }^{f}$ Institut für Kernphysik, Universität zu Köln, D-50937, Köln, Germany \\ E-mail: ornelas@cii.fc.ul.pt
}

In this work we present the results from the analysis of the experimentally measured angular distributions of the reaction ${ }^{106} \mathrm{Cd}(\alpha, \alpha){ }^{106} \mathrm{Cd}$ at several different energies around the Coulomb barrier. The difficulties that arise in the study of ${ }^{106} \mathrm{Cd}-\alpha$-nuclear potential and the so called Family Problem are addressed in this work.

11th Symposium on Nuclei in the Cosmos

19-23 July 2010

Heidelberg, Germany.

\footnotetext{
*Work supported by FCT grant PTDC/FIS/103902/2008, ERC StG No. 203175 and OTKA NN83261 (EuroGENESIS)

${ }^{\dagger}$ Speaker.
} 


\section{Introduction}

The story of nucleosynthesis beyond the iron peak is that of neutron capture reactions in the astrophysical $s$ and $r$-process. However, there are 35 stable isotopes located in the proton-rich side whose production cannot be explained in the framework of the slow and rapid neutron capture process. One of the most accepted mechanisms for the synthesis of the so-called $p$-nuclei is based on photodisintegration reactions on neutron-rich seed nuclei $[1,2]$. The reaction rates sensitivity to the nuclear input in a $p$-process network calculation has been considered $[1,3]$. Whereas the low mass $p$-nuclei $(70 \leq \mathrm{A} \leq 120)$ show a stronger dependence on the $(\gamma, \mathrm{p})$ reaction rates, the heavy mass stable proton-rich isotopes are very sensitive to variations on the $(\gamma, \alpha)$ reaction rates, which directly depend on the considered $\alpha$-nucleus potential.

By measuring the angular distribution of alpha particles at energies close above the Coulomb barrier it is possible to extract information on the $\alpha$-nucleus potential. However, as shown for ${ }^{144} \mathrm{Sm}$ [4] and ${ }^{92} \mathrm{Mo}$ [5], at these energies several "families" of the potential (characterized by different depths of the real nuclear potential) provide an equally good description of the scattering data.

Here we present the first results obtained in the analysis of the potential families extracted from the angular distribution of the reaction ${ }^{106} \mathrm{Cd}(\alpha, \alpha){ }^{106} \mathrm{Cd}[6]$ measured at the ATOMKI laboratory at energies around the Coulomb barrier. The sensitivity of the families to the description of $\alpha$ particle capture reactions measured close to $p$-process relevant energies [7] is also shown.

\section{Systematic Study: The family problem}

The analysis of the elastic scattering cross section of the reaction ${ }^{106} \mathrm{Cd}(\alpha, \alpha){ }^{106} \mathrm{Cd}$ was performed within the optical model:

$$
U(r)=V_{C}(r)+V_{R} f\left(r, R_{R}, a_{R}\right),+i W_{V} f\left(r, R_{V}, a_{V}\right)+i W_{S} g\left(r, R_{S}, a_{S}\right)
$$

with the Coulomb potential $\left(\mathrm{V}_{C}\right)$ :

$$
V_{C}(r)= \begin{cases}Z_{P} Z_{T} e^{2 \frac{3-\left(\frac{r}{R_{C}}\right)^{2}}{2 R_{C}}} & \text { for } r<R_{C} \\ Z_{P} Z_{T} \frac{e^{2}}{r} & \text { for } r \geq R_{C}\end{cases}
$$

and a complex nuclear potential, for which we adopted different volume and surface parameterizations of the Woods-Saxon form $V(r)=V_{0} f_{V}(r)$ and $W(r)=W_{V} f_{V}(r)+W_{S} g\left(r, R_{S}, a_{S}\right)$ with $f_{i}=\left(1+\exp \left(\frac{r-R_{i}}{a_{i}}\right)\right)^{-1}, R_{i}=r_{i} A^{1 / 3}, i=V, S$ and $g\left(r, R_{S}, a_{S}\right)=-4 a_{S} \frac{d f_{S}(r)}{d r}$ as described in $[8,9,10]$. The optical model is designed to simplify the interaction between two nuclei, such that an otherwise difficult to solve many-body problem is simplified to the interaction between two inert nuclei using an effective potential.

The main goal of this work was to obtain the potential that accurately describes the measured crosssection data. As shown in previous works $[4,5]$ there are several potential families, corresponding to different depths of the real part of the nuclear potential, that describe with similar quality the elastic scattering data at energies around the Coulomb barrier. Following a similar approach we have performed a systematic variation of the real part of the nuclear potential studying the angular 
distribution measured at the highest energy $\left(\mathrm{E}_{\text {lab }}=19.6 \mathrm{MeV}\right)$ in order to obtain all the families of the ${ }^{106} \mathrm{Cd}-\alpha$ potential. We adopted the code a0 [11] to iteratively calculate the angular distribution for each potential. In our approach, the diffuseness $\left(\mathrm{a}_{r}\right)$ and radius $\left(\mathrm{R}_{r}\right)$ of the real part of the potential were fixed, while the real depth potential $\left(\mathrm{V}_{0}\right)$ was successively decreased and the imaginary potential adjusted for each case. The results are presented in Figure 1. We obtained 14 different potentials that equally describe the ${ }^{106} \operatorname{Cd}(\alpha, \alpha){ }^{106} \mathrm{Cd}$ interaction, i.e, their $\chi^{2} / F$ is very similar (lower part of the figure). The variations of the volume integrals for both real $\left(\mathrm{J}_{R}\right)$ and imaginary $\left(\mathrm{J}_{I}\right)$ parts of the potential are shown as well. As indicated, all families describe with similar quality

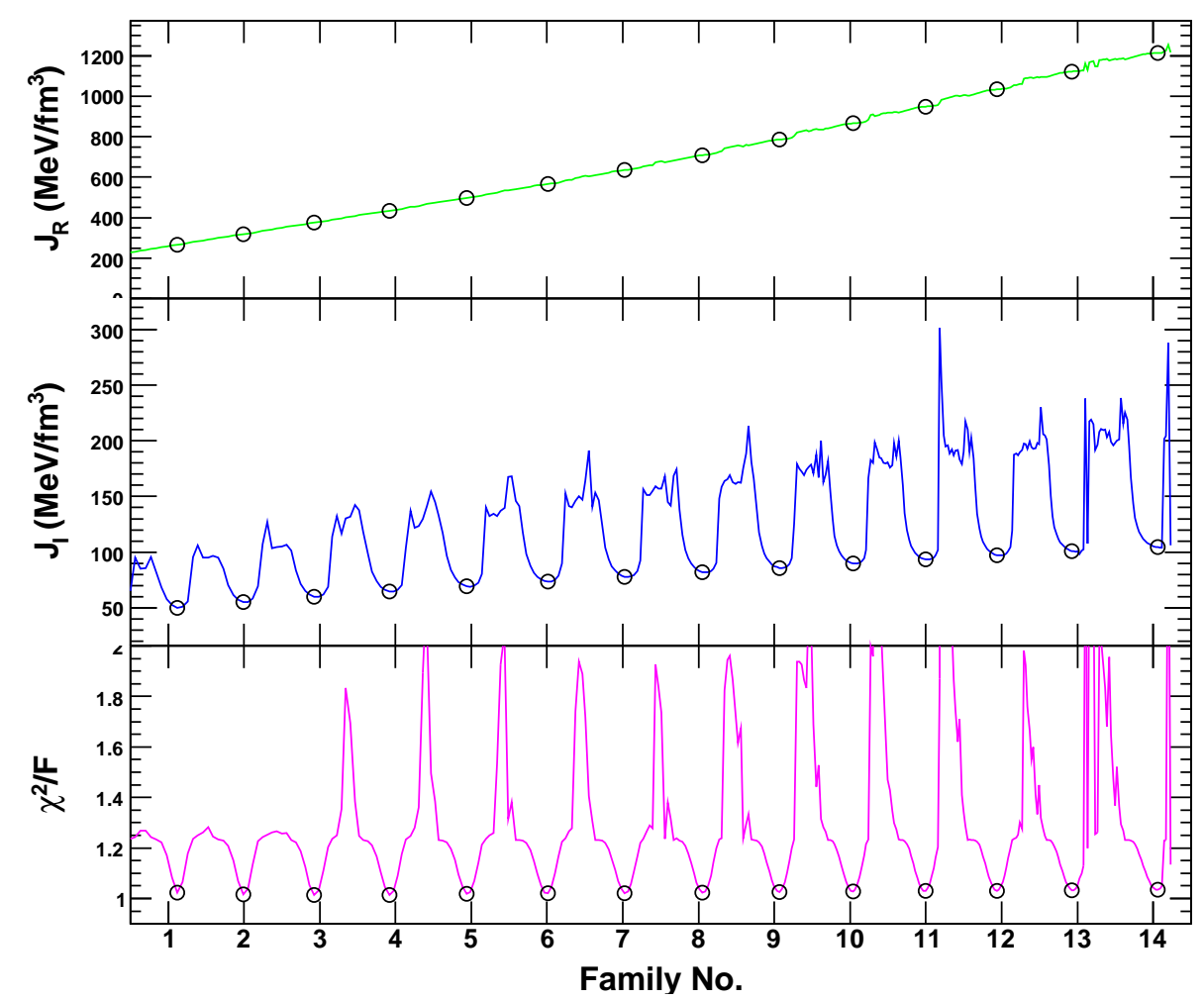

Figure 1: Obtained $\chi^{2} / F$ (bottom) for the different families of the potential extracted from the analysis of the ${ }^{106} \mathrm{Cd}(\alpha, \alpha){ }^{106} \mathrm{Cd}$ reaction at $\mathrm{E}_{l a b}=19.6 \mathrm{MeV}$. The resulting volume integrals for the real $\left(\mathrm{J}_{R}\right.$, top) and imaginary $\left(\mathrm{J}_{I}\right.$, center) potentials are shown as well.

the experimental results. We present as an example Family No. 6 in Figure 2 together with the experimental data for the three considered angular distributions [6].

The 14 different potentials that we encountered are a consequence of the family problem that arises from the fact that the scattering cross section is only sensitive to the asymptotic properties of the wave function (reflexion coefficients $\eta_{L}$ and phase shifts $\delta_{L}$ ).

\section{Description of existing capture data}

Once finished the analysis of the elastic scattering data, the obtained families of the potential 

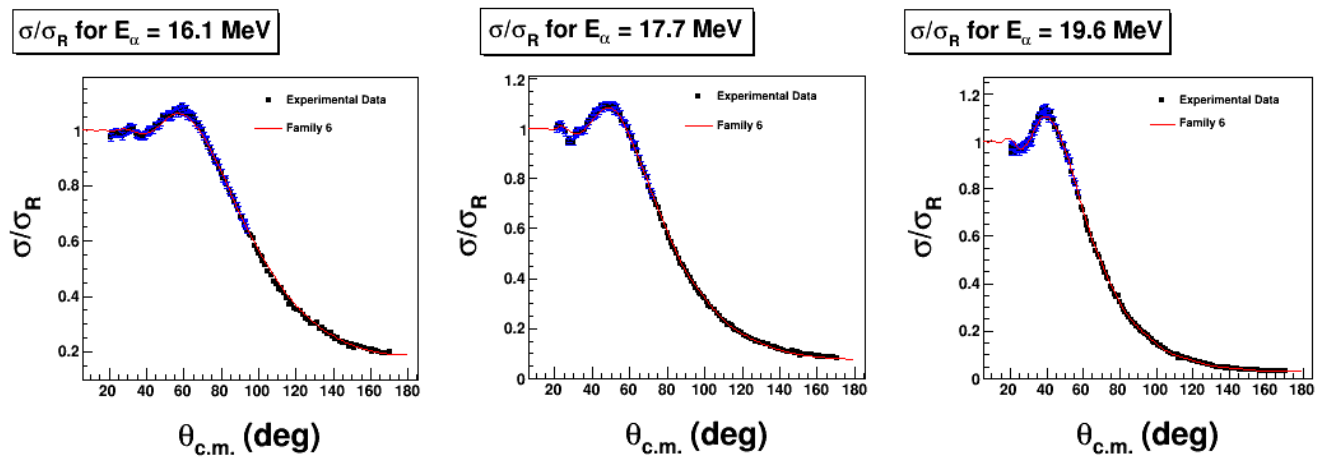

Figure 2: Experimental Rutherford normalized angular distributions for the reaction ${ }^{106} \mathrm{Cd}(\alpha, \alpha){ }^{106} \mathrm{Cd}$ at $\mathrm{E}_{l a b}=16.1,17.7$ and $19.6 \mathrm{MeV}$ [6]. In addition, the results obtained using Family No. 6 are shown. All three angular distributions are accurately described by the extracted families.

were used to calculate the cross section for the processes ${ }^{106} \mathrm{Cd}(\alpha, \gamma){ }^{110} \mathrm{Sn},{ }^{106} \mathrm{Cd}(\alpha, \mathrm{n}){ }^{109} \mathrm{Sn}$, and ${ }^{106} \mathrm{Cd}(\alpha, \mathrm{p}){ }^{109}$ In. For this purpose, the NON-SMOKER ${ }^{W E B}$ [12] application was employed.

The resulting astrophysical S-factors are presented in Figure 3 together with the experimental data from [7] .

As it can be observed, all derived potentials overestimate the experimental data for ${ }^{106} \operatorname{Cd}(\alpha, \gamma)^{110} \mathrm{Sn}$ and ${ }^{106} \mathrm{Cd}(\alpha, n){ }^{109} \mathrm{Sn}$ processes, but describe the ${ }^{106} \mathrm{Cd}(\alpha, \mathrm{p}){ }^{109} \mathrm{In}$ reasonably well. More remarkable is the fact that all families of the potential, despite the large range of the nuclear potential depths for both real and imaginary parts, provide almost the same output. This indicates that the real part of the potentials derived in this work is very insensitive in the description of radiative capture of $\alpha$ particles by the nucleus ${ }^{106} \mathrm{Cd}$.
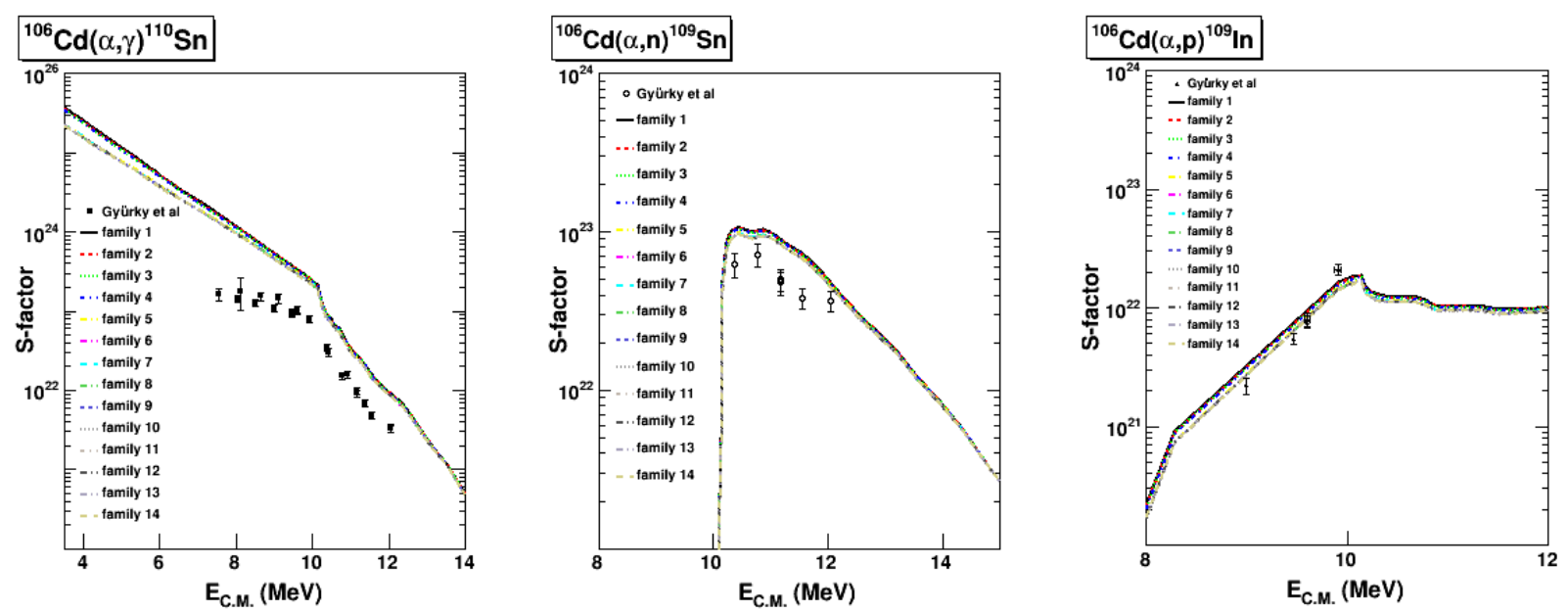

Figure 3: Astrophysical S-factor of the ${ }^{106} \mathrm{Cd}(\alpha, \gamma){ }^{110} \mathrm{Sn},{ }^{106} \mathrm{Cd}(\alpha, \mathrm{n}){ }^{109} \mathrm{Sn}$ and ${ }^{106} \mathrm{Cd}(\alpha, \mathrm{p}){ }^{109} \mathrm{In}$ reactions together with the results obtained from the 14 different potential families obtained in this study. 


\section{Conclusions}

In this work we have performed a systematic study of the potential families of the system $\alpha$ $-{ }^{106} \mathrm{Cd}$ analyzing the angular distribution of the reaction ${ }^{106} \mathrm{Cd}(\alpha, \alpha){ }^{106} \mathrm{Cd}$ at energies around the Coulomb barrier. We obtained 14 different potential parameterizations, which were used in the calculation of the astrophysical S-factor for the ${ }^{106} \mathrm{Cd}(\alpha, \gamma){ }^{110} \mathrm{Sn},{ }^{106} \mathrm{Cd}(\alpha, \mathrm{n}){ }^{109} \mathrm{Sn}$, and ${ }^{106} \mathrm{Cd}(\alpha, p){ }^{109}$ In reactions. An almost equal result was obtained in describing the compound reactions at energies of astrophysical interest. Further investigations are underway to clarify the sensitivity of the real and imaginary parts of the nuclear potential in the description of these processes and will be the topic of a dedicated paper.

\section{References}

[1] T. Rauscher. Branchings in the gamma process path revisited. Phys. Rev. C, 73(1),015804, 2006.

[2] M. Arnould and S. Goriely. The p-process of stellar nucleosynthesis: astrophysics and nuclear physics status. Phys. Rep., 384,1, 2003.

[3] W. Rapp, J. Görres, M. Wiescher, H. Schatz, and F. Käppeler. Sensitivity of p-Process Nucleosynthesis to Nuclear Reaction Rates in a 25 M Supernova Model. Astrophys. J., 653,474, 2006.

[4] P. Mohr, T. Rauscher, H. Oberhummer, Z. Máté, Z. Fülöp, E. Somorjai, M. Jaeger, and G. Staudt. ${ }^{144} \mathrm{Sm}-\alpha$ optical potential at astrophysically relevant energies derived from ${ }^{144} \mathrm{Sm}(\alpha, \alpha){ }^{144} \mathrm{Sm}$ elastic scattering. Phys. Rev. C, 55,1523, 1997.

[5] Z. Fülöp, G. Gyürky, Z. Máté, E. Somorjai, L. Zolnai, D. Galaviz, M. Babilon, P. Mohr, A. Zilges, T. Rauscher, H. Oberhummer, and G. Staudt. ${ }^{92} \operatorname{Mo}(\alpha, \alpha){ }^{92}$ Mo scattering, the ${ }^{92}$ Mo $-\alpha$ optical potential, and the ${ }^{96} \mathrm{Ru}(\gamma, \alpha){ }^{92}$ Mo reaction rate at astrophysically relevant energies. Phys. Rev. C, 64(6),065805, Nov 2001.

[6] D. Galaviz. Systematic study of $\alpha$-nucleus potentials for neutron-deficient nuclei and it's astrophysical applications. PhD thesis, Technischen Universität Darmstadt, 2004.

[7] G. Gyürky, G. G. Kiss, Z. Elekes, Z. Fülöp, E. Somorjai, A. Palumbo, J. Gorres, H. Y. Lee, W. Rapp, M. Wiescher, N. Özkan, R. T. Guray, G. Efe, and T. Rauscher. Alpha-induced cross sections of ${ }^{106}$ Cd for the astrophysical p process. Phys. Rev. C, 74(2),025805, 2006.

[8] M. Avrigeanu, W. von Oertzen, A. J. M. Plompen, and V. Avrigeanu. Optical model potentials for alpha-particles scattering around the coulomb barrier on A 100 nuclei. Nucl. Phys., A723,104, 2003.

[9] M. Avrigeanu, W. von Oertzen, and V. Avrigeanu. On temperature dependence of the optical potential for alpha-particles at low energies. Nucl. Phys., A764,246, 2006.

[10] M. Avrigeanu, A. Obreja, F. Roman, V. Avrigeanu, and W. von Oertzen. Complementary optical-potential analysis of $\alpha$-particle elastic scattering and induced reactions at low energies. Atomic Data and Nuclear Data Tables, 95,501 - 532, 2009.

[11] H. Abele and P. Mohr. code a0_ version 1.50, 2010. (unpublished).

[12] T. Rauscher and F.-K. Thielemann. Astrophysical reaction rates from statistical model calculations. At. Data Nucl. Data Tab., 75,1-352, 2000. 